\title{
Therapeutic results of patients with endometriosis and ovarian cancer
}

\author{
Corresponding author: \\ Elżbieta Stasienko \\ Clinical Department \\ of Gynaecological Oncology, \\ The Franciszek Lukaszczyk \\ Oncology Centre \\ Romanowskiej $2 \mathrm{St}$. \\ 85-796 Bydgoszcz, Poland \\ Tel.: +48535535045 \\ E-mail: elzbieta.stasienko@gmail.com
}

Medical Research Journal 2016 Volume 1, Number 3, 105-107 10.5603/MRJ.2016.0018

Copyright (C) 2016 Via Medica ISSN 2451-2591

\begin{abstract}
Introduction. The aim of this study is to compare the therapeutic results of patients with ovarian cancer coexisting with endometriosis with a group of patients with cancer formed in the endometriosis focus in the scar after caesarean section.

Methods. We analysed the therapeutic results of patients with ovarian cancer coexisting with endometriosis with a group of patients with cancer formed in the endometriosis focus in the scar after caesarean section. They were operated in the Oncology Centre in Bydgoszcz. All the patients had endometriosis and ovarian cancer confirmed in one tissue preparation.

Results. The probability of death at any time is 7.826-times higher in the group with scar cancer than in the group with endometriosis and ovarian cancer.

Conclusions. Because scar cancer that arises in the background of endometriosis is an extremely rare condition and also because the prognosis for this group of patients is poor we consider it necessary to plan combination therapy by a multidisciplinary oncological team.

Key words: ovarian cancer, endometriosis, ovarian neoplasms
\end{abstract}

Med Res J 2016; 1 (3): 105-107

\section{Introduction}

Uncontrolled divisions of cells, the formation of implants in adjacent tissue, stromal destruction, the ability to spread to distant organs, similar risk factors, and oestrogen-dependent growth - this resemblance between endometriosis and cancer initiated on-going studies that are aimed at determining the relationship between the two diseases [1].

Malignant transformation associated with the ectopic location of endometrial tissue concerns mainly the ovaries $(70 \%)$, and only about $25 \%$ of such cancers arise out of the pelvis. The proliferative process located in the scar after prior laparotomy is especially rare, with 37 cases described in the literature. However, given the growing number of caesarean sections, it is believed that cancers in this location will become more frequent.

Although retrograde menstruation occurs in $70 \%$ of menstruating women, not all of them will develop endometriosis. Therefore, the thesis has been put forward that individual characteristics of the immune system are responsible for the effective elimination or specific tolerance towards the development of endometrial tissue outside the uterus. Hence, this disease can be the result of ineffective elimination of endometrial cells from the peritoneal cavity.

\section{Methods}

Recruitment of subjects involved a search through the electronic database of patients treated at the Oncology Centre in Bydgoszcz. The coexistence of endometriosis and ovarian cancer in one postoperative tissue specimen, confirmed in histological examination, was an inclusion criterion. Preparations, which were prepared in a standard manner, fixed in formalin, embedded in paraffin, stained with haematoxylin and eosin, were subject to histological reassessment, which confirmed endometriosis-associated ovarian carcinoma. Endometriosis and ovarian cancer were defined according to World Health Organisation guidelines.

Fifty-three patients receiving surgical treatment at the Oncology Centre in Bydgoszcz were included in the study.

\section{Results}

The analysis performed using the Cox model shows that the analysed groups differ significantly with regard to survival (because the $p$ value is below 0.05). HR for the group with scar tissue cancer 
Table 1. 1-year, 3-year and 5-year survival rates and the median survival for each analysed group

\begin{tabular}{|c|c|c|c|c|c|c|c|c|c|c|}
\hline \multirow[t]{2}{*}{ Group } & \multirow{2}{*}{$\begin{array}{c}\text { Number } \\
\text { of patients }\end{array}$} & \multirow{2}{*}{$\begin{array}{c}\text { Number } \\
\text { of events } \\
\text { (deaths) }\end{array}$} & \multicolumn{3}{|c|}{ Survival } & \multirow{2}{*}{$\begin{array}{l}\text { Median } \\
\text { survival } \\
\text { (months) }\end{array}$} & \multirow[t]{2}{*}{ HR } & \multicolumn{2}{|c|}{ 95\% Cl for $\mathrm{HR}$} & \multirow[t]{2}{*}{$\mathbf{p}$} \\
\hline & & & 1-year & 3-year & 5-year & & & & & \\
\hline $\begin{array}{l}\text { Endometriosis } \\
\text { and ovarian } \\
\text { cancer }\end{array}$ & 46 & 13 & $89.13 \%$ & $75.45 \%$ & $75.45 \%$ & $\begin{array}{l}>\text { the longest } \\
\text { follow-up }\end{array}$ & Ref. & & & \\
\hline $\begin{array}{l}\text { Scar tissue } \\
\text { cancer in } \\
\text { endometriosis }\end{array}$ & 7 & 4 & $51.43 \%$ & $25.71 \%$ & $25.71 \%$ & 18.25 & 7.826 & 2.062 & 29.703 & $p=0.003$ \\
\hline
\end{tabular}

$\mathrm{Cl}$ - confidence interval

is 7.826 , i.e. in this group the probability of death at any time is 7.826-times higher than in the group with endometriosis and ovarian cancer (Tab. 1). It can also be observed on Kaplan-Meier curves: a curve for the group with scar tissue cancer is located lower and it indicates a poorer prognosis (Fig. 1). The table can also be used to read 1-year, 3-year and 5-year survival rates and the median survival for each analysed group. For example, in the group with scar tissue cancer 5 -year survival was $25.71 \%$, i.e. $25.71 \%$ of patients in this group survived for at least 5 years. In the group with scar tissue cancer the median survival was 18.25 months, i.e. half of the patients in this group died after this time. The median survival in the group with endometriosis and ovarian cancer is longer than the duration of the longest follow-up period. It means that more than half of patients were alive after the end of the follow-up period in this group (this can be observed on a chart: the Kaplan-Meier curve does not reach 50\%).

\section{Discussion}

Numerous studies have shown that the creation of endometriosis is related to the reduced activity of NK cells and/or macrophages [2, 3] which comprise macrophages, B cells, T cells, natural killer (NK). However, other works have demonstrated that the reduced efficiency of the immune system against autologous endometrial cells is not a simple relation $[4,5]$.

The characteristics of the tumour cell were described in 2011 by Hanahan and Weinberg [6]. This cell should have its own mechanisms of resistance against apoptosis, the ability to form nourishing blood vessels, invade neighbouring tissues, and create satellite metastatic lesions, as well as be insensitive to the anti-proliferative signals of the body and have a certain genetically conditioned instability that allows for still new growth opportunities. Because OSE (ovarian epithelium surface) has pluripotent properties, e.g. the ability to undergo cyclic EMT (epithelial to mesenchymal transition) in the process of "healing" after ovulation, the hypothesis of frequent carcinogenesis appeared in this place [7].

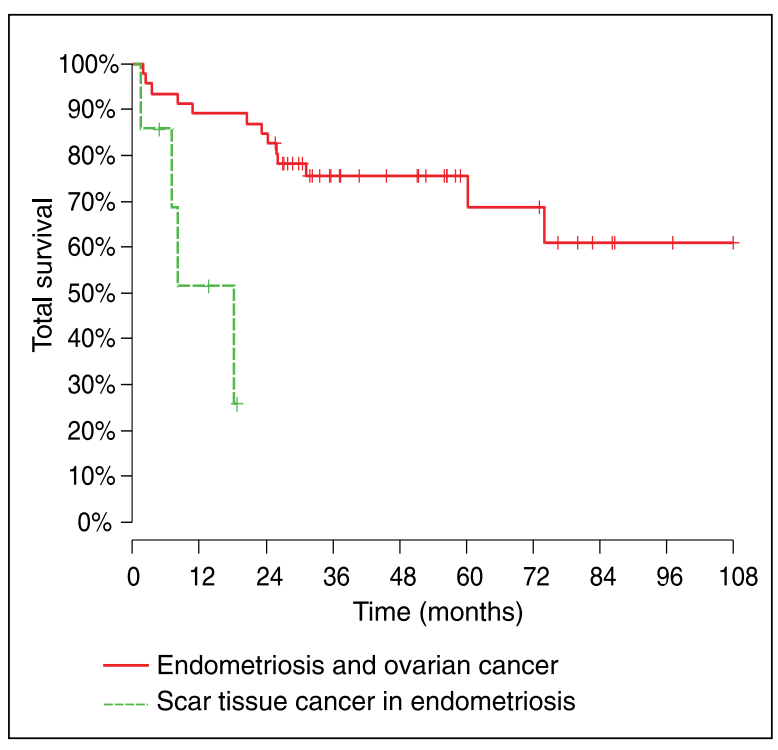

Figure 1. Kaplan-Meier curves: the curve for the group with scar tissue cancer is located lower, and it indicates a poorer prognosis

As noted by many researchers, the presence of ovarian endometriosis creates a new microenvironment with certain disorders in the cytokine and hormonal OSE profile. These disorders would favour the passage of neoplastic transformation. Assuming that endometriosis promotes the formation of the compounds with the properties of growth factors for cancer cells and causes the existence of a chronic inflammatory effect, over time it increases the chance of accumulation of mutations during the process of DNA transcription and repair. Balkwill and Mantovani emphasise the role of chronic inflammation in the course of carcinogenesis by Virchow, who as early as in the nineteenth century observed that chronic inflammation promoted the formation of certain types of cancers [8]. Uncontrolled, excessive cell proliferation causes the response from the tumour cell microenvironment by producing chemotactic substances aimed at mobilising macrophages and monocytes [9]. Alluvial macrophages releasing growth factors (VEGF-A, VEGF-C, TNF, HGF, EGF, PDGF), and 
chemokines (CXCL12 and IL-8) exacerbate inflammation [10]. It is emphasised that the role of macrophages infiltrating the tumour is not clear. It has been observed that stimulation of the tumour development in certain circumstances may be responsible for its destruction [11]. Most studies indicate, however, that growth and pro-inflammatory factors, which are produced by macrophages, promote welfare of the tumour to a greater degree than induction of the effective antitumor immune response [8]. It has been demonstrated that they play an important role in the formation of satellite changes and induction of the metastatic stage of tumour. Having proteolytic activity, they initiate the stage of microinvasion of stroma by tumour cells through degradation of the basement membrane [12]. In their work, Mantovani et al. [13] showed that the activation of tumour-associated macrophages (TAMs) is an essential step in the initiation of metastasis.

Causing abnormal cytokine and hormonal milieu ovarian endometriosis approaches toward the expression profile, which is typical of cancer of this organ. Persistent and over again exacerbated inflammation, which is caused by the presence of ectopic endometrial cells, produces the specific microenvironment with many cells and even more intracellular signals. Clonal proliferation of endometrial cells in the development of ectopic endometrial tissue has to create the microenvironment conditioning and supporting this change. The microenvironment with at least part of an ovarian tissue will be influenced by the same factors that guarantee clonal, ectopic, and autonomous growth. The multiplicity of divisions, accompanying DNA repairs and chronic inflammation, which is always connected with ectopic endometrial changes, facilitates the accumulation of mutations as an unrecoverable error inevitably leading to malignant transformation. The induction of persistent inflammation, which is a characteristic of endometriosis, has local and distant systemic consequences [14]. Increased expression of proinflammatory chemokines IL-1, IL-6, IL-8 and TNF- $\alpha$ in the course of ongoing inflammation has a major impact on the stability of the endometrial tissue in ectopic locations [15].

Chronically-stimulated immune effector cells regulate intracellular signal transduction cascades by the transcription factor $\mathrm{NF}_{\kappa} \mathrm{B}$, which is a certain link between carcinogenesis and inflammation [16]. On the other hand, chronically activated immune cells inhibit the anti-tumour response acquired by the immune system continuously during smouldering of inflammation. $\mathrm{NF} \kappa \mathrm{B}$ controls many processes necessary for cancer to achieve autonomy, such as resistance to apoptosis, neoangiogenesis, independence in growth signals, stromal invasion, and formation of metastases [6]. The direct participation of $\mathrm{NF} \kappa \mathrm{B}$ in the formation of metastases was described by Luo et al. [17]. A decrease in the expression of the nuclear factor resulted in the reduction of tumour growth driven by inflammation. Furthermore, it has been shown that this factor is activated not only by the stimulant effect from the microenvironment, but also by the genetic background [18].

\section{Conclusions}

Due to the extremely poor prognosis for patients with scar cancer coexisting with endometriosis we consider it necessary for a multidisciplinary oncological team to plan combination therapy. We do believe that radiotherapy is an essential part of adjuvant treatment.

\section{References}

1. Munksgaard PS, Blaakaer J. The association between endometriosis and ovarian cancer: a review of histological, genetic and molecular alterations. Gynecol Oncol 2012; 124: 164-169.

2. Giudice LC. Endometriosis. NEJM 2010; 362: 2389-2398.

3. Ho HN, Wu MY, Yang YS. Peritoneal cellular immunity and endometriosis. Am J Reprod Immunol 1997; 38: 400-412.

4. Montgomery GW, Nyholt DR, Zhao ZZ et al. The search for genes contributing to endometriosis risk. Hum Reprod Update 2008; 14: 447-457.

5. Daraï E, Detchev R, Hugol D, Quang NT. Serum and cyst fluid levels of interleukin (IL)-6, IL-8 and tumour necrosis factor-alpha in women with endometriomas and benign and malignant cystic ovarian tumours. Hum Reprod 2003; 18: 1681-1685.

6. Hanahan D, Weinberg RA. Hallmarks of cancer: the next generation. Cell 2011; 144: 646-674.

7. Ahmed N, Thompson EW, Quinn MA. Epithelial-mesenchymal interconversions in normal ovarian surface epithelium and ovarian carcinomas: an exception to the norm. J Cell Physiol 2007; 213: 581-588.

8. Balkwill F, Mantovani A. Inflammation and cancer: back to Virchow? Lancet 2001; 357: 539-545.

9. Robinson SC, Coussens LM. Soluble mediators of inflammation during tumor development. Adv Cancer Res 2005: 93: 159-187.

10. Allavena P, Sica A, Garlanda C, Mantovani A. The Yin-Yang of tumor-associated macrophages in neoplastic progression and immune surveillance. Immunol Rev 2008; 222: 155-161.

11. Mantovani A, Bottazzi B, Colotta F, Sozzani S, Ruco L. The origin and function of tumor-associated macrophages. Immunol Today 1992; 13: $265-270$.

12. Pollard JW. Tumour-educated macrophages promote tumour progression and metastasis. Nat Rev Cancer 2004; 4: 71-78

13. Mantovani A, Schioppa T, Porta C, Allavena P, Sica A. Role of tumor-associated macrophages in tumor progression and invasion. Cancer Metastasis Rev 2006; 25: 315-322.

14. Ness RB, Grisso JA, Cottreau $C$ et al. Factors related to inflammation of the ovarian epithelium and risk of ovarian cancer. Epidemiology 2000; 11: 111-117

15. Nezhat F, Datta MS, Hanson V, Pejovic T, Nezhat C, Nezhat C. The relationship of endometriosis and ovarian malignancy: a review. Fertil Steril 2008; 90: 1559-1570

16. Lee D-F, Kuo H-P, Chen C-T et al. IKK beta suppression of TSC1 links inflammation and tumor angiogenesis via the mTOR pathway. Cell 2007; 130: 440-455.

17. Luo J-L, Maeda S, Hsu L-C, Yagita H, Karin M. Inhibition of NF-kappaB in cancer cells converts inflammation-induced tumor growth mediated by TNFalpha to TRAIL-mediated tumor regression. Cancer Cell 2004; 6: 297-305

18. Karin M. Nuclear factor-kappaB in cancer development and progression. Nature 2006; 441: 431-436. 